\section{PO-0754 NEUROENDOCRINE REGULATION OF RETINOIC ACID RECEPTORS DURING LUNG BRANCHING IN NORMAL AND HYPOPLASTIC LUNGS}

${ }^{1}$ P Pereira Terra, ${ }^{1} \mathrm{RS}$ Moura, ${ }^{1} \mathrm{C}$ Nogueira-Silva, ${ }^{2} \mathrm{~J}$ Correia-Pinto. ' $S$ Surgical Sciences Research Domain, ICVS/3B'S, Braga, Portugal; '2Pediatric Surgery, Hospital de Braga, Braga, Portugal

\subsection{6/archdischild-2014-307384.1393}

The pathogenesis of pulmonary hypoplasia and congenital diaphragmatic hernia $(\mathrm{CDH})$ is unknown. $\mathrm{CDH}$ represents a spectrum of lung hypoplasia and consequent pulmonary hypertension $(\mathrm{PH})$ that leads to high morbidity and mortality of patients. We studied neuroendocrine factors and retinoic acid in order to achieve the relation underlying them.

At 13.5 days post-conception normal and $\mathrm{CDH}$ lungs were cultured in vitro during four days with DMSO, retinoic acid, bombesin, ghrelin, bombesin antagonist and ghrelin antagonist. Morphometric analysis were done after the culture and Western Blot (WB) was performed to quantify the protein levels of retinoic acid receptors (RAR). Immunohistochemestry (IHC) was performed as well on normal, nitrofen and CDH E17.5 lungs.

When compared with controls, $\mathrm{CDH}$ lungs presented higher expression of RAR in IHC and WB. Moreover in normal lungs after the administration of bombesin and ghrelin the expression of RAR also increases and in case of retinoic acid administration it decreases. Regarding bombesin and ghrelin antagonists administration, RAR expression decreases as it was expected. In terms of morphometry, treated groups showed an increase in branching, perimeter and area.

This study, shows for the first time that retinoic acid deficit on $\mathrm{CDH}$ lungs is associated with neuroendocrine factors overexpression. Furthermore, neuroendocrine factors such as ghrelin and bombesin sensitise for RAR expression.

\section{PO-0755 IN VIVO MIR-200B AS A POTENTIAL THERAPY FOR CONGENITAL DIAPHRAGMATIC HERNIA}

${ }^{1}$ P Pereira Terra, ${ }^{2} \mathrm{~N}$ Khoshgoo, ${ }^{3} \mathrm{~B}$ Iwasiow, ${ }^{1} \mathrm{~J}$ Correia-Pinto, ${ }^{3} \mathrm{R}$ Keijzer. ${ }^{1}$ Surgical Sciences Research Domain, ICVS/3B'S, Braga, Portugal; ${ }^{2}$ Department of Physiology, Manitoba Institute of Child Health, Winnipeg, Canada; ${ }^{3}$ Department of Surgery, Manitoba Institute of Child Health, Winnipeg, Canada

\subsection{6/archdischild-2014-307384.1394}

Congenital diaphragmatic hernia $(\mathrm{CDH})$ is associated with lung hypoplasia and pulmonary hypertension (PH) leading to a high morbidity and mortality. MicroRNAs, affect gene expression and miR-200b is involved in epithelial-mesenchymal transition in cancer and is downregulated in rat nitrofen lungs.

We hypothesised that miR-200b regulates lung branching. Therefore, we aimed to evaluate whether in vivo administration of miR-200b influences lung development and branching morphogenesis.

Timed-pregnant dams were treated with nitrofen $(\mathrm{CDH}$ group) or olive oil (control group) on $\mathrm{E} 9$ and received a tail vein injection of miR-200b mimics $(5 \mathrm{mg} / \mathrm{kg}$ ) or saline, respectively. At E21.5 and P0, we dissected the lungs and evaluated the presence or absence of $\mathrm{CDH}$. We estimated the lung hypoplasia and we did histological studies to determine radial alveolar count (RAC) and medial arterial thickness.

Nitrofen lungs treated with miR-200 b mimics, in vivo display improved development and larger size. These embryos were also bigger than the embryos of the nitrofen group plus saline. Their size was similar to control embryos. After RAC analysis revealed that nitrofen treated lungs have larger alveolar spaces than nitrofen lungs. In terms of arteries we did not observe any differences.

Administration of miR-200b in vivo decreases lung hypoplasia and increases the size of the lungs as well as alveolar airspaces. These data show promissing results for miR-200b as a potential therapeutic target in $\mathrm{CDH}$ patients.

- This work was supported by grants from the $\mathrm{MICH}$, MMSF, Molly Towell, GFT surgeons and Thorlakson Foundation.

\section{PO-0756 CRICOID PRESSURE AS AN ADJUNCT TO NEONATAL INTUBATION: A NATIONAL SURVEY}

RF Power, JFA Murphy. Neonatology, National Maternity Hospital, Dublin, Ireland

\subsection{6/archdischild-2014-307384.1395}

Background and aims Although widely documented for use in adult intubation, there is paucity of literature available on the application of cricoid pressure (CP) during neonatal intubation. It is briefly mentioned in the NRP guideline, as a possible adjunct measure. This study was mounted to determine how widely the technique is used by paediatricians and neonatologists in clinical practice.

Methods A questionnaire was devised, consisting of eight questions. The questionnaire was distributed nationally to 40 consultant paediatricians/neonatologists, 31 specialist registrars, 40 neonatal nurses, midwives and ANNPs.

Results The overall response rate was 76\% $(\mathrm{n}=84)$. Findings summarised in table 1

\begin{tabular}{ll} 
Abstract P0-0756 Table 1 & Cricoid survey findings \\
\hline Parameter & No. Respondents $(\mathbf{n}=\mathbf{8 4})(\%)$ \\
\hline Perform intubation & $54(64.3)$ \\
Assist in intubation & $29(36)$ \\
Familiar with CP & $82(97.6)$ \\
Feel that CP improves visualisation of the glottis & $70(83.3)$ \\
Feel that CP aids opening of the vocal cords & $16(19)$ \\
Feel that CP facilitates intubation & $40(47.6)$ \\
Aware of CP complications & $63(75)$ \\
\hline
\end{tabular}

Conclusions - Almost all the healthcare professionals surveyed were aware of the cricoid pressure technique.

- The majority felt that cricoid pressure has a role in improving glottis visualisation.

- One half found that it facilitated intubation.

- A minority felt that it helped to open the vocal cords during intubation.

- The high response rate provides an accurate reflection of neonatal intubation practice in Ireland.

\section{PO-0757 PERINATAL FACTORS IN THE DEVELOPMENT OF BRONCHOPULMONARY DYSPLASIA}

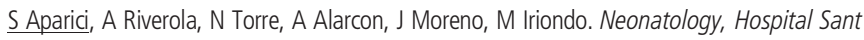
Joan de Déu, Barcelona, Spain

10.1136/archdischild-2014-307384.1396 